The $8^{\text {th }}$ International Conference of the Slovenian Society for Non-Destructive Testing

"Application of Contemporary Non-Destructive Testing in Engineering"

September 1-3, 2005, Portorož, Slovenia, pp. 27-33

\title{
CLASSIFICATION OF ULTRASONIC SIGNALS
}

\author{
V. Matz, M. Kreidl, R. Šmíd \\ Czech Technical University, Faculty of Electrical Engineering, department of Measurement, \\ Technická 2, Prague 6, 166 27, \\ E-mails: matzv@feld.cvut.cz,kreidl@feld.cvut.cz,smid@feld.cvut.cz
}

\begin{abstract}
In ultrasonic defectoscopy it is very difficult to detect flaw in materials with coarse-grain structure. The ultrasonic signals measured on these materials contain echoes which are very similar to fault echo. These echoes arise from grains which are contained in material. For detection of flaw various methods for suppressing of echoes from grains have to be used. In this work we used the method for filtering of ultrasonic signal based on discrete wavelet transform. For classification of ultrasonic signals in A-scan we used pattern recognition method called support vector machines. In this study we classify signals with fault echo, echo from weld and back-wall echo. Ultrasonic signals were measured on material used for constructing airplane engines. The experimental results indicate the performance of the proposed approach.
\end{abstract}

Keywords: Ultrasonic testing, Discrete wavelet transform, Support vector machines

\section{Introduction}

Ultrasonic non-destructive testing is part of non-destructive evaluation which offers to detect the undersurfaces flaws in the materials. The basic principle of the ultrasonic testing is ultrasound which uses the transmission of high-frequency sound waves in a material to detect a discontinuity or to locate changes in material properties. The sound is introduced into a material and the reflections (echoes) are received from internal imperfections or from surface of the part. The received signal consists of echoes from flaw or surface and echoes from scattering of coarse grained structure of material. Noise, formed from scattering of inhomogeneous micro-structures has to be cancelled. Several techniques have been proposed to reduce noise from signal. Split spectrum processing, FIR filtering and discrete wavelet transform were studied and the best method for filtering of ultrasonic signal were searched [1]. Among them discrete wavelet transform gives very good results.

This paper introduces an experimental study involving measurement of ultrasonic signals with consequent analysis and signal processing. Proposed processing of ultrasonic signals allows efficient filtering by which it is possible to reduce the noise formed from scattering and electronic circuitry.

The first section describes the filtering of ultrasonic signal and introduces the basic analysis. For filtering method based on the discrete wavelet transform called wavelet packets [2] is used.

In the next section a method for classification based on support vector machines is introduced. The method support vector machines is based on the maximum of the minimal length between 
support vectors which characterizes the features. Features are based on extraction of the basic mathematical operations as is root mean square, standard deviation or mean value.

In the final section system for classification of ultrasonic signals is described. With the system it is possible to classify the three different types of ultrasonic: clear signal, signal with flaw and signal from weld.

\section{Classification of ultrasonic signals}

\subsection{System for measurement}

For classification of ultrasonic signals high frequency ultrasonic system was used. For measurement of ultrasonic signals the transducer with operation frequency of $20 \mathrm{MHz}$ was used. The frequency $20 \mathrm{MHz}$ is the best compromise for detection of flaw in coarse grainy material. For measurement the two types of materials (steel and coarse grainy material) were used. Steel and coarse grainy materials were welded. The ultrasonic signals were measured in three different places: place without flaw, place with flaw and in the centre of the weld.

\subsection{Filtering of ultrasonic signal}

Pre-processing of ultrasonic signals contains the amplitude normalization and filtering. For filtering of ultrasonic signal a method based on the discrete wavelet transform (DWT) called wavelet packets (WP) is used. This method is very efficient in the time domain. The ultrasonic signal in A-scan is measured and WP is used for the improvement of the signal-to-noise ratio. The wavelet transform is a multiresolution analysis technique that can be used to obtain the timefrequency representation of the ultrasonic signal. Filtering procedure is based on decomposition of signal using DWT in $\mathrm{N}$ levels using band pass filtering and decimation to obtain the approximation and detail coefficients. Next step is thresholding of detail coefficients and reconstruction of signal from detail and approximation coefficients using inverse transform (IDWT). The WP method is a generalization of wavelet decomposition that offers a larger range of possibilities for signal analysis. In wavelet analysis, a signal is split into an approximation and detail coefficients. The approximation is then itself split into a second-level approximation and detail, and the process is repeated. In WP analysis, the detail coefficients as well as the approximation coefficients can be splited. Hard thresholding was used for thresholding detail coefficients. Hard thresholding can be described as the process of setting to zero the elements whose absolute values are lower than the threshold. For thresholding of detail coefficients the local threshold value based on standard deviation was used [3]:

$$
T h r=k \cdot \sqrt{\frac{1}{N-1} \cdot \sum_{i=1}^{N}\left(D c_{i}-\bar{D} c\right)^{2}},
$$

where $\quad k \quad$ is coefficient related to crest factor of filtered signal (crest factor is the ratio of the peak value to the RMS value),

Dc are detail coefficients at each level,

$N \quad$ is length of each set of detail coefficients.

Usually global threshold is used in WP filtering. In our study the local thresholding was used instead of global thresholding. The threshold is computed from detail coefficients at each level of decomposition. Then the computed threshold is used for thresholding detail coefficients at the same level.

For examination of quality of filtering signal-to-noise ratio is computed: 


$$
S N R=20 \cdot \log \left(\frac{S_{e f}}{N_{e f}}\right)[\mathrm{dB}],
$$

where

$S_{e f} \quad$ is the root mean square value of the noisy part of the raw signal.

$F_{e f} \quad$ is the root mean square value of an adequate part of the filtered signal.

\subsection{Support vector machines}

The basic theory of SVM used in this application is first presented details can be found elsewhere [4]. The SVM maximizes margin between classes which increases generalization ability. The classification problem can be restricted to consideration of the two class problem without loss of generality. In this problem the goal is to separate the two classes by a function which is induced from available problem. The goal is to produce a classifier that will work well on unseen examples. The basic idea is linear classifier that maximizes the distance between separating hyperplane and the nearest data point of each class.

Consider the problem of separating the set of training vectors belonging to two separate classes,

$$
\left(x_{1}, y_{1}\right), \ldots \ldots \ldots,\left(x_{l}, y_{l}\right), x \in R^{n}, y \in\{-1,1\} \text {, }
$$

with hyperplane,

$$
\langle w, x\rangle+b=0
$$

The set of vectors is said to be optimally separated by the hyperplane if it is separated without error and the distance between the closest vectors to the hyperplane is maximal. The parameters $w, b$ in equation (4) are constrained by,

$$
\min _{i}\left|\left\langle w, x^{i}\right\rangle+b\right|=1
$$

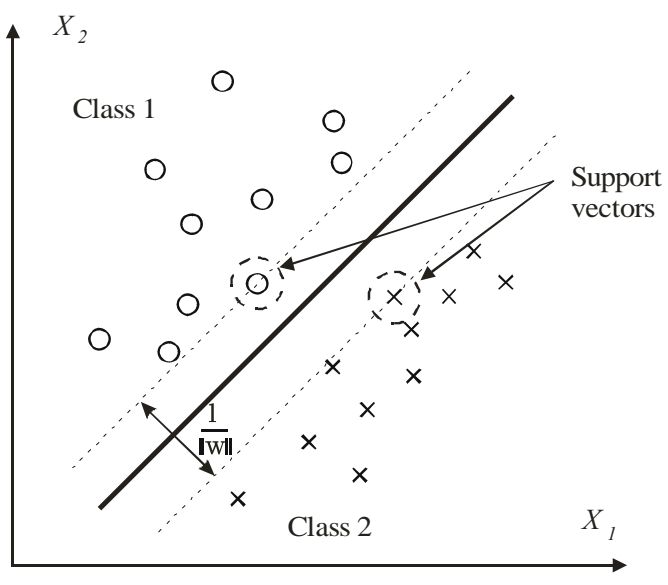

Fig. 1: SVM Linear classifier.

The distance $d(w, b ; x)$ of a point $\mathrm{x}$ from the hyperplane $(w, b)$ is,

$$
d(w, b ; x)=\frac{\left|\left\langle w, x^{i}\right\rangle+b\right|}{\|w\|} .
$$


The aim is to convert the problem into a formulation without constraints. Lagrange function $L$ is introduced, $\alpha_{i}$ Lagrange multipliers,

$$
L\left(w, b, \alpha_{i}\right)=\frac{1}{2}\|w\|^{2}-\sum_{i=1}^{L} \alpha_{i}\left(w^{T} x_{i}\right) y_{i}+\sum_{i=1}^{L} \alpha_{i}
$$

Once the Lagrange multipliers for the optimal hyperplane have been determined the following separating rule can be used by expressing the optimal weight vector in terms of support vectors and the Lagrange multipliers:

$$
f(\vec{x})=\operatorname{sign}\left(\sum_{\text {support vectors }} y_{i} \alpha_{i}^{0}\left(\vec{x}_{i}^{T} \cdot \vec{x}\right)+b_{0}\right),
$$

where $\vec{x}_{i}$ are the support vectors, $\alpha_{i}^{0}$ are the corresponding Lagrange coefficients, and $b_{0}$ is the threshold constant.

A Support Vector Machine maps the input space into a high dimensional feature space and then constructs an optimal hyperplane in the feature space.

\subsection{Feature extraction}

For the feature extraction the characteristic properties of ultrasonic signal have to be used. From the shape of ultrasonic signal which was measured on different place contains the fault and backwall echo, only the backwall echo or the signal from the center of weld the basic amplitude characteristics were derived. The basic features include:

$$
\begin{aligned}
\text { mean value: } & A V G=\frac{1}{N} \sum_{i=1}^{N} x_{i}, \\
\text { root mean square value: } & E F C=\sqrt{\frac{1}{N} \sum_{i=1}^{N} x_{i}^{2}}, \\
\text { standard deviation: } & S T D=\sqrt{\frac{1}{N-1} \sum_{i=1}^{N}\left(x_{i}-A V G\right)^{2}}, \\
\text { absolute value: } & A B S=\sum_{i=1}^{N}\left|x_{i}\right| .
\end{aligned}
$$

For the calculation of descriptive features the back-wall echo was cut off. The average, root mean square a standard deviation value were computed from the back-wall echo and from the rest of the signal. The signal which contains only the backwall echo has the smallest average value but in the opposite the signal measured in the centre of the weld has the higher average value because the signal in the centre of the weld contains more echoes which were caused by scattering of the signal.

The features of ultrasonic signal were put into the SVM classifier and the hyperplane was computed.

\section{Experimental results}

For measurement of ultrasonic signals two different materials (steel and coarse grained metallic material) were used. In all materials artificial flaws were created, and two parts of these materials were welded. In case of coarse grain material which is used for construction of airplane engines 
the structure of material is inhomogeneous and the measured ultrasonic signal contains the echoes from scattering from grains.

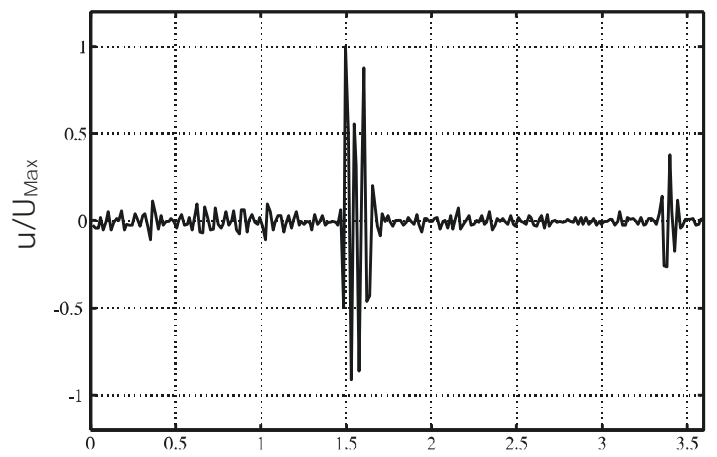

a)

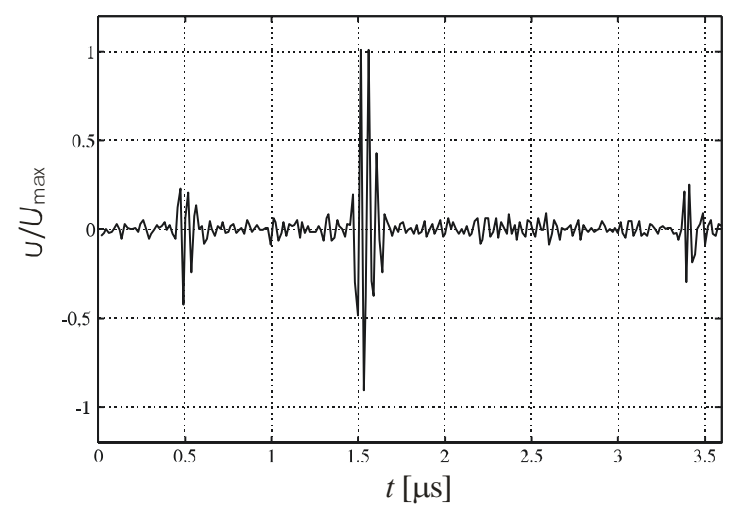

c)

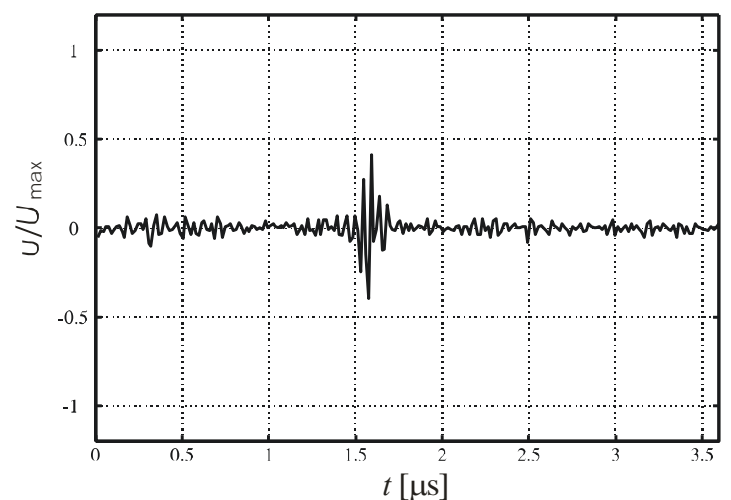

b)
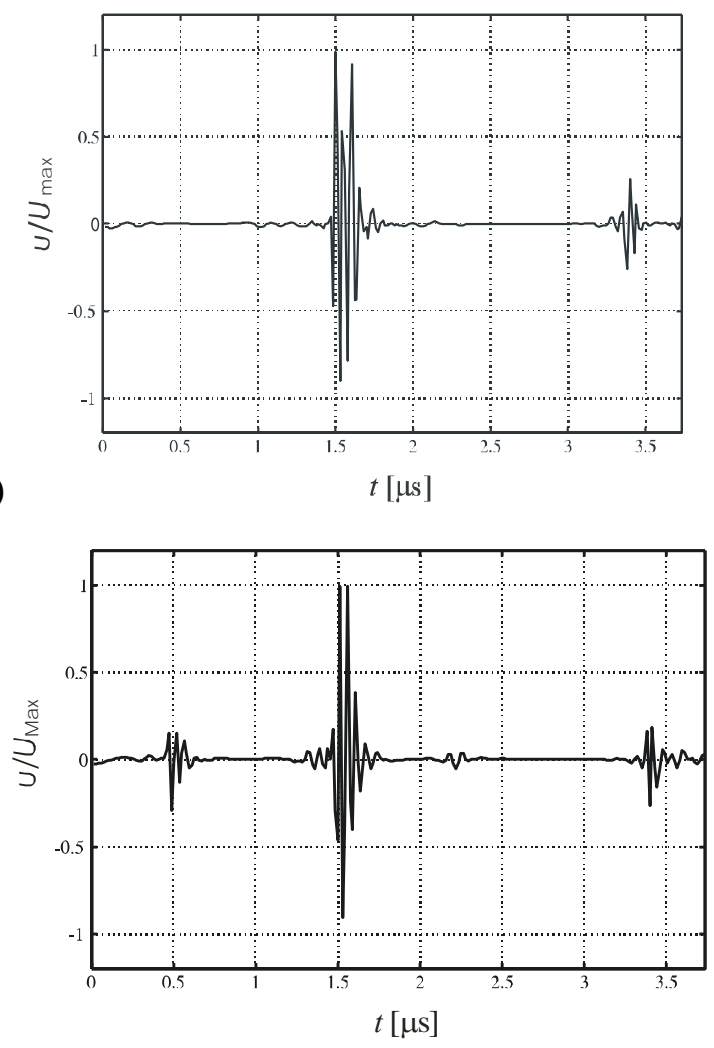

d)

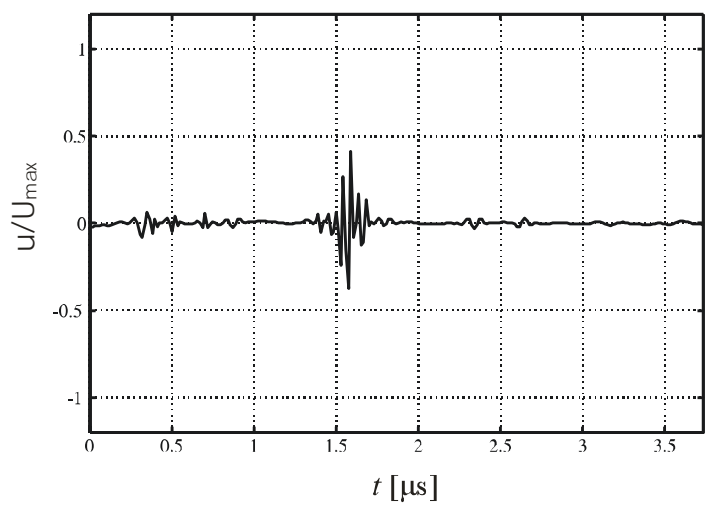

Fig. 2: Ultrasonic signal of grainy material - a) clear raw signal, b) filtered signal, c) raw signal with fault echo, d) filtered signal with fault echo, e) raw signal measured on weld, f) filtered signal measured on weld.

For filtering of ultrasonic signals the wavelet packets method was used. In this method the local threshold was used. It means that the threshold was computed in every detail coefficients and these coefficients were thresholded. This method gives the highest suppressing of background noise which is contained in measured signal. Noise reduction in the depicted case for WP is $S N R=17[\mathrm{~dB}]$. The results of measured and filtered ultrasonic signals are in Fig. 2.

For the successful classification the informative features should be obtained. The features were computed from the amplitude characteristics and were mapped to the three classes. The class 1 is related to the signal without flaw, the class 2 to the place with the flaw and the class 3 to the weld. For the training the different algorithm of support vector machines functions as Successive overrelaxation (SOR), one-against-one decomposition (OAO), one-against-all decomposition (OAA) and sequential minimal optimizer (SMO) were used. The different functions were 
combined with the different features. The results of different features and functions are illustrated in Fig. 3.

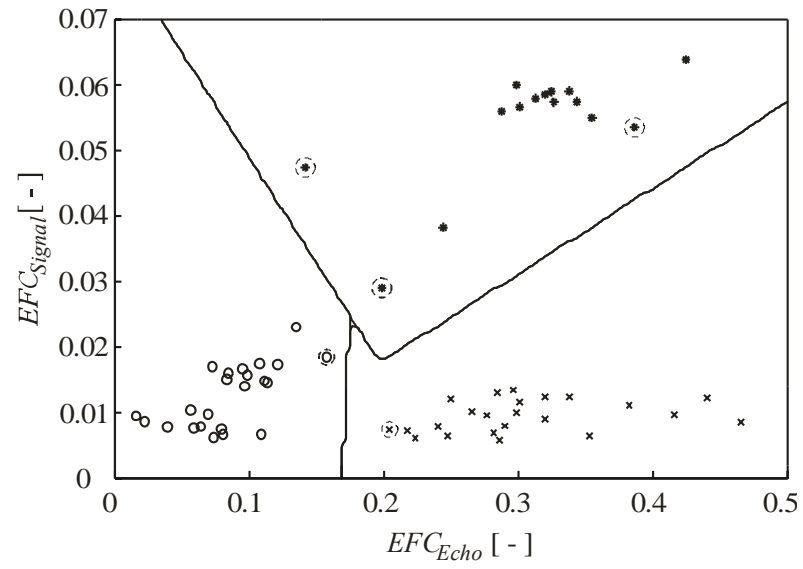

a)

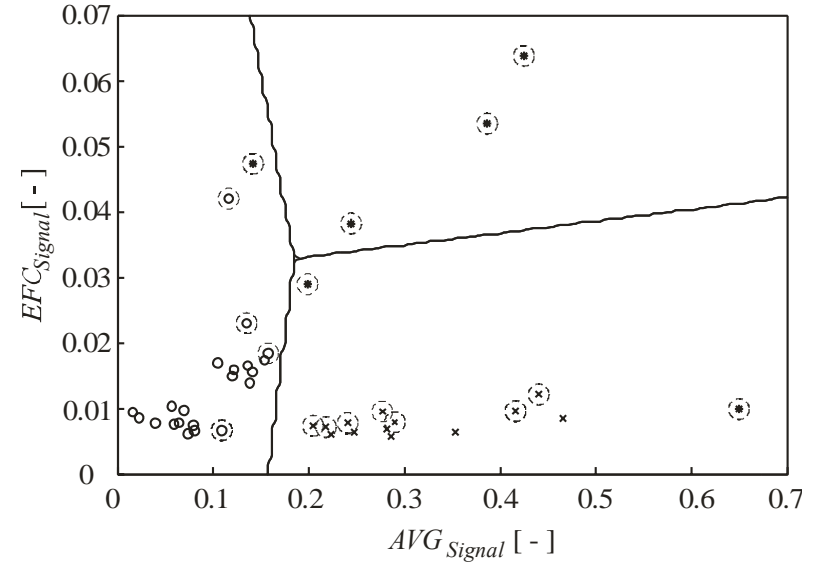

c)

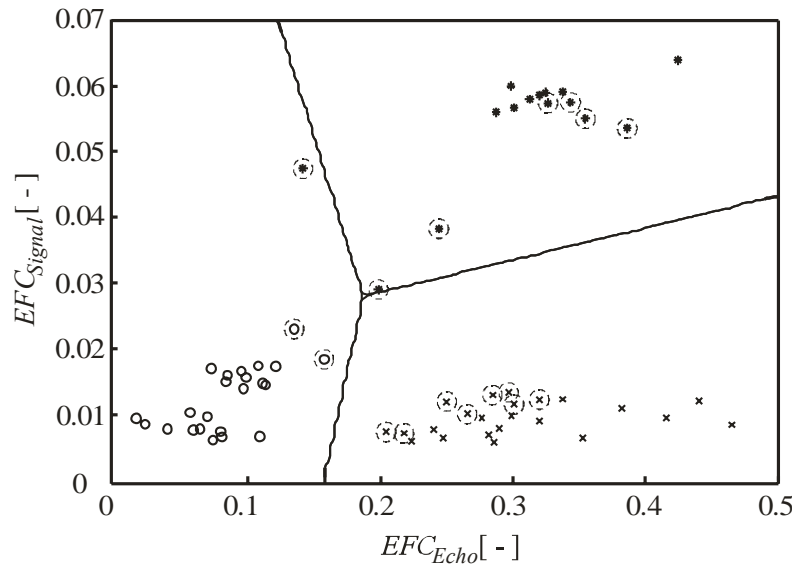

b)

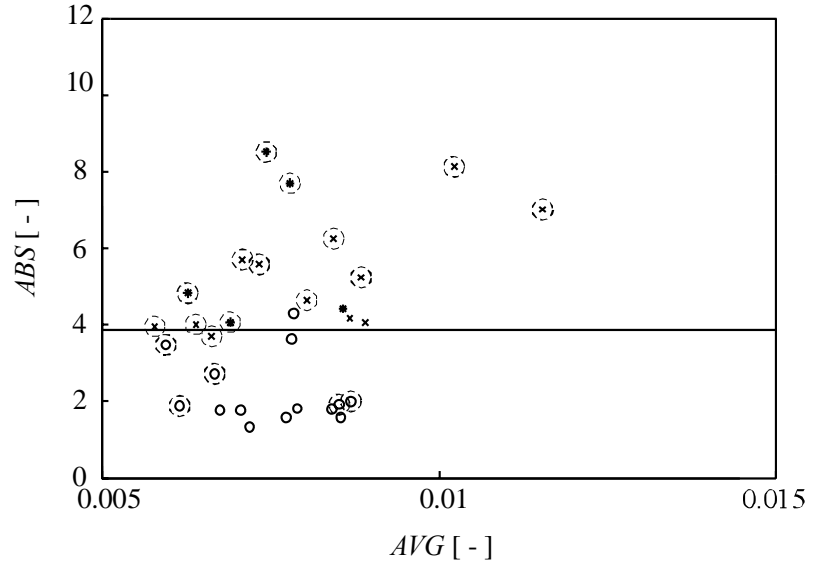

d)

Fig. 3: Feature spaces with different functions and features - a) OAO, b) SOR, c) OAA, d) SMO.

The best results were obtained with one-against-one decomposition with features of root mean square value of echo and rest of signal. All the three classes were separated and the performance of classification was $100 \%$ (error rate $0 \%$ ). The proposed algorithm for the classification of ultrasonic signals is efficient in ultrasonic defectoscopy for automated detection and classification of flaws.

\section{Conclusions}

In this paper a new method for the classification of ultrasonic signals is proposed. In the ultrasonic signals measured on materials with coarse grain structure it is not possible to easily recognize the flaws. In this case the method for filtering of ultrasonic signals based on the discrete wavelet transform called wavelet packets was used. This method offers efficient filtering of ultrasonic signals. For the classification of ultrasonic signals the support vector machines was used. With this method it is possible to classify the signal with back-wall echo, signal with fault echo and signal measured on weld. The proposed method is useful for the automated classification of ultrasonic signals in industry. 


\section{Acknowledgements}

The research of "Classification of ultrasonic signals" was supported by the research program No. MSM6840770015 "Research of Methods and Systems for Measurement of Physical Quantities and Measured Data Processing " of the CTU in Prague sponsored by the Ministry of Education, Youth and Sports of the Czech Republic.

\section{References}

[1] Ericsson, L., Stepinski, T.: Algorithms for suppressing ultrasonic backscattering from material structure, Ultrasonics, Volume 40, issue 1-8, pages 733-734, May 2002.

[2] Paul S Addison: The Illustrated Wavelet Transform Handbook: Introductory Theory and Applications in Science, Engineering, Medicine and Finance. Napier University, Edinburgh, UK, 2004, ISBN 0750306920.

[3] Matz V., Kreidl, M., Šmíd, R.: Signal-to-Noise Ratio Improvement based on the Discrete Wavelet Transform in Ultrasonic Defectoscopy. Acta Polytechnica. 2004, vol. 44, no. 4, s. 61-66. ISSN 1210-2709.

[4] Gunn, S., R.: Support Vector Machines for Classification and Regression. Technical Report, University of Southampton, 1998.

[5] Vapnik, V.: Statistical learning theory. Wiley, Chichester, GB, 1998. 